\title{
IDENTIFICATION AND CHEMICAL CHARACTERIZATION OF
}

\section{AZADIRACHTA INDICA LEAF EXTRACTS THROUGH THIN LAYER CHROMATOGRAPHY}

\author{
M. Mukunda Vani ${ }^{1}$, P. Sagar Suresh Rao ${ }^{2}$, G. Nikhilesh Varma ${ }^{3}$, K.Pushpitha ${ }^{4}$, Linisha Biswal ${ }^{\mathbf{5}}$ \\ 1,2,3,4,5 Department of Chemical Engineering, Anurag Group of Institutions, Hyderabad, India. \\ vanivikram@yahoo.com, sagarpopshetwar42@gmail.com
}

\begin{abstract}
In this article, extraction of Azadirachta indica compounds has been done using solvents namely ethanol, methanol, benzene, ethyl acetate, toluene which are widely used in industries as solvents. All the compounds present in Azadirachta indica leaves are supposed to be soluble in solvents but this work has been done to show that different solvents have different capacity to extract the compounds. The semi quantitative analysis has been done with the help of thin layer chromatography (TLC) which shows the efficiency of every individual solvent to extract compounds from Azadirachta indica. Thin layer chromatography can physically separate the compounds from pure extracts hence this method is preferred for analysis purpose. Even composition and their respective percentage can be concluded using various analysis method. It is helpful to analyse the solubility of different compounds of Azadirachta indica in particular solvent.
\end{abstract}

Keywords: Extraction, Thin Layer Chromatography (TLC), Semi Quantitative Analysis, Solubility Of Compounds, Characterization And Identification

\section{INTRODUCTION}

Plants are the human best friend providing natural valuable herbal medicine for curing various ailments [1]. Azadirachta indica is a tree which is over loaded with number compounds that can be used for medicinal purposes [3]. Nimbidin, a major crude bitter principle extracted from the leaves has several biological activities. From this crude principle some tetranortriterpenes, including nimbin, nimbinin, nimbidinin, nimbolide and nimbidic acid, ascorbic acid, amino acid, Azadirachtin, etc have been isolated. But separation and identification of these compounds is more important which can be studied through this article. This article provides precise information on the identification of nimbin, ascorbic acid, amino acid and Azadirachtin. Hence Azadirachta indica is preferred for the study.

Selection of solvents is based on the factor of solubility which is widely used over industries [2, 4]. All the solvents used in this study are of pure pharmaceutical grade to enhance the quality of research work.

Large number of solvents can be used to extract the compounds present in Azadirachta indica but efficiency and semi-qualitative analysis can be seen with the help of thin layer chromatography. Thin layer chromatography is a method which can physically separate the compounds from extract $[3,5]$.

Semi quantitative analysis of the compounds of Azadirachta indica dissolved in each solvent has been studied and identification of the compounds were done using TLC analysis [7, 9].

\section{MATERIALS AND METHOD:}

\subsection{Extraction From Azadirachta Indica Leaves:}

Juvenile and mature leaves of Azadirachta indica tree were plucked from campus of Anurag Group of Institutions, Hyderabad. These leaves were washed with distilled water and air dried at room temperature. $50 \mathrm{~g}$ of dried leaves were macerated in $100 \mathrm{ml}$ of pure solvent and extract was collected after 48 hours and two more consecutive extracts from residue has been collected using same method. The collected extracts were then cold centrifuged to remove suspended material and the supernatant was oven dried at temperature corresponding to the boiling point of the solvent used. These extracts were stored at $4{ }^{\circ} \mathrm{C}$ in the refrigerator for further use [16]. The different solvents used are ethanol, methanol, benzene, ethyl acetate and toluene.

\subsection{Thin Layer Chromatography Analysis:}

TLC is a primary; easy to use analytical method and solvent used are un-hazardous with no requirement of sophisticated instruments [3]. Usually it is composed of stationary phase and mobile phase, which are performed on a sheet of solid surface such as glass, plastic, aluminium foil that is coated with absorbent material such as silica powder, aluminium oxide and cellulose, which is called as stationary phase [3, 4].

Mobile phase may consist of single or mixture of solvents depending on extracts to separate. This mobile phase is drawn up through the stationary phase by capillary action allowing separation of various compounds on the basis of 
their solubility and retardation in stationary phase and mobile phase $[3,7]$. TLC principle works on a solubility rule "Like Dissolves Like" and is followed on separation of mixture of polar, non polar, mid polar compounds from the extracts on a static phase[11,12]. The compounds that are not as much soluble in mobile phase will have an affinity for stationary phase and will travel to a smaller extent than the soluble compounds. An $\mathrm{R} f$ value is "retardation factor" or "ratio to front" which can be calculated using the formula [8].

$$
R f=\frac{\text { distance travelled by compound }}{\text { distance travelled by solvent front }}
$$

These Rf values can be calculated by observing spots on TLC plates under UV trans-illuminator at $365 \mathrm{~nm}$. An $\mathrm{R} f$ value occurs between $0-1$ and depends upon following factors, which determine the efficiency of a chromatographic separation $[8,9]$.

Depending upon the compounds in a solvent tract the choice of a solvent system is made. Water (polar) is universal solvent dissolving most of the compounds in it. Chloroform is non polar. Ethyl's acetate is mid polar. Separating chamber is usually allowed to get saturated with vapours of solvent / mobile phase [21, 23]. The phenomenon of like dissolves like is applied here. Compound that is polar will be dissolved in polar solvent and compound that is non polar will be dissolved in non polar solvent system and most of the compound, which are polar and non polar have an ability to get dissolved in mid polar solvent such as ethyl acetate. Sometime combinations of mobile phase are used to separate polar, non-polar, mid polar compounds [17, 19].

\subsection{Selection Of Solvent System For TLC:}

Selection of solvent system can be done on the capacity to isolate the maximum number of fluorescent spots which indicates presence of those many compounds in crude extracts.

Several TLC solvent trials were used such as Acetic acid: Ethanol (1:3); Acetic acid: Water (1:10); Ethyl acetate: Ethanol (1:3); Hexane: Ethyl acetate (1:1); Methanol: Toluene (8:2). Among these, Hexane: Ethyl acetate (1:1) was the best as it was able to separate 11 spots from the crude ethanol extract and Methanol: Toluene (8:2) solvent exhibits the second best as it was able to separate 5 spots from the crude extract.

\section{RESULTS AND DISCUSSIONS:}

Since large number of useful compounds are present in Azadirachta indica and these compounds can be separated through various methods like distillation, extraction, etc. This study is done on basis of solvent extraction using different types of solvents widely used over industries. The quantitative analysis of different solvent extracts of Azadirachta indica was done with the help of Thin layer chromatography analysis. This is one of the useful method for characterization and identification of compounds present in Azadirachta indica leaves.

TLC analysis was performed for all solvent extracts of Azadirachta indica and results of the present investigation are as follows. According to TLC analysis, if RF value of pure component is matching to any RF value of extract shows the presence of that pure compound in extract.

The standard RF values of Nimbin, Ascorbic acid, Amino acid, Azadirachtin are 0.09, 0.74, 0.62, 0.70 respectively which are obtained from literature [1] are shown in table 1. Table 2 shows the TLC analysis of methanol extract using solvent system hexane: ethyl acetate (5:5) and methanol: toluene $(8: 2)$ as the solvent system. The spots of different extracts observed under UV light as shown in figure 1 and 2 indicated the same RF values which shows the presence of above mentioned compounds in the extract. TLC analysis for benzene, ethyl acetate, ethanol and toluene extracts using Hexane-Ethyl acetate (5:5) and Methanol-Toluene (8:2) as the solvent system are tabulated in tables $3,4,5$ and 6 respectively.

From all these, it was observed that alcohol as solvent could extract more number of compounds from Azadirachta indica and this was spotted under UV light after performing TLC analysis using hexane: ethyl acetate as solvent system .
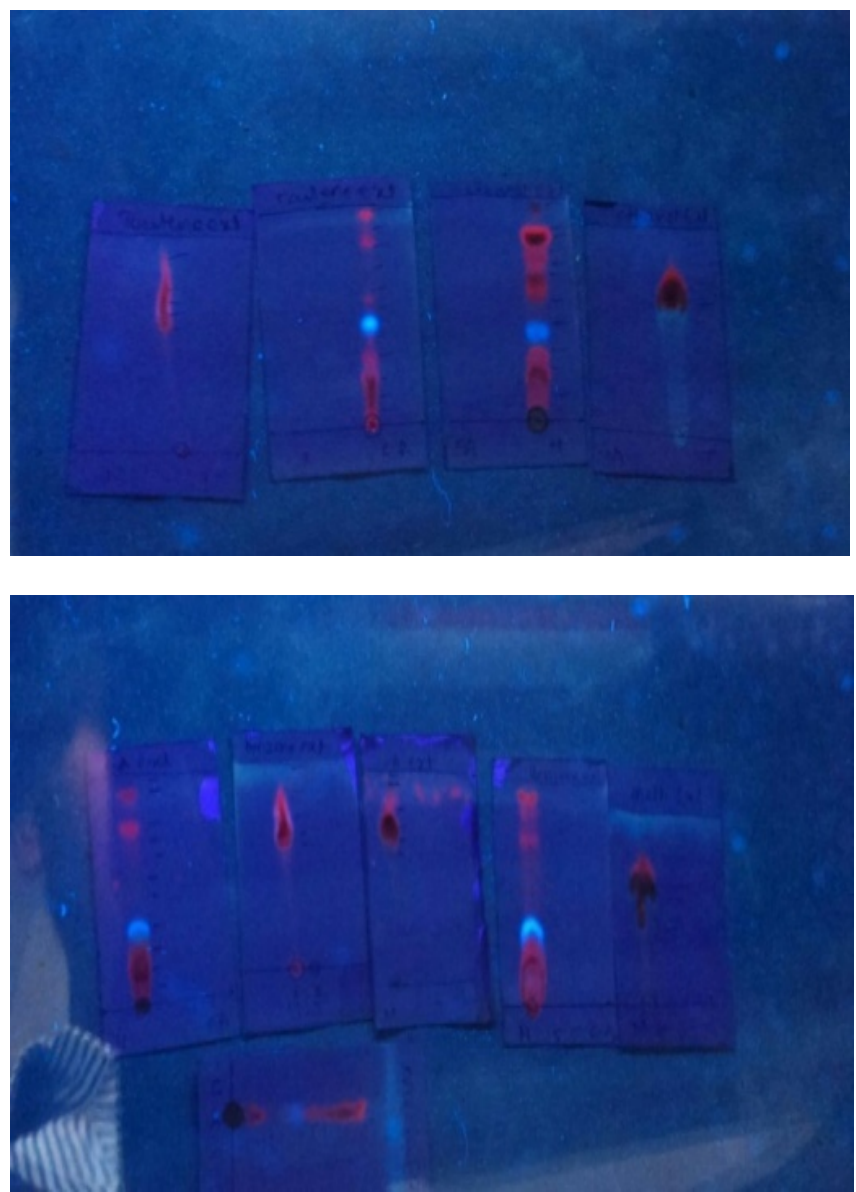

Fig.1: TLC plates of different extracts under UV light 
Thin Layer Chromatography of Azadirachta indica leaf extracts and their Rf values:

Table: 1Standard Rf values

\begin{tabular}{|c|c|c|c|c|c|}
\hline \multicolumn{3}{|c|}{ Solvent: Hexane: Ethyl acetate(5:5) } & \multicolumn{3}{c|}{ Solvent: Methanol: Toluene(8:2) } \\
\hline \multicolumn{2}{|c|}{ Nimbin } & UV light & Rf & Visible light & UV light \\
\hline Rf & Visible light & Blue & 0.45 & Light green & Pink \\
\hline 0.09 & - & Blue & 0.51 & Green & Pink \\
\hline 0.91 & - & & & \\
\hline \multicolumn{2}{|c|}{ Ascorbic acid } & UV light & & & \\
\hline Rf & Visible light & Pink & & & \\
\hline 0.74 & - & UV light & & & \\
\hline \multicolumn{2}{|c|}{ Amino acid } & Pink & & & \\
\hline Rf & Visible light & & & & \\
\hline 0.62 & - & UV light & & & \\
\hline \multicolumn{2}{|c|}{ Azadirachtin } & Light green & & & \\
\hline Rf & Visible light & & & & \\
\hline 0.7 & -
\end{tabular}

Table: 2Methanol extract

\begin{tabular}{|c|c|c|c|c|c|c|c|}
\hline \multicolumn{4}{|c|}{ Solvent: Hexane-Ethyl acetate $(5: 5)$} & \multicolumn{4}{|c|}{ Solvent: Methanol- Toluene( $8: 2)$} \\
\hline \multicolumn{4}{|c|}{ Material } & \multicolumn{4}{|c|}{ Material } \\
\hline $\mathrm{Rf}$ & Visible light & UV light & $\begin{array}{l}\text { Compound } \\
\text { identified }\end{array}$ & $\mathrm{Rf}$ & Visible light & UV light & $\begin{array}{c}\text { Compound } \\
\text { identified }\end{array}$ \\
\hline 0.056 & - & - & & 0.48 & Light green & Pink & Nimbin \\
\hline 0.064 & - & - & & $\mathbf{0 . 5 3}$ & green & Pink & Nimbin \\
\hline $\mathbf{0 . 1 0}$ & - & Blue & Nimbin & 0.625 & - & Dark & \\
\hline 0.20 & Light green & Dark & & 0.678 & - & - & \\
\hline 0.35 & Light green & Pink & & 0.89 & - & - & \\
\hline 0.46 & Light green & Pink & & & & & \\
\hline 0.45 & - & Pink & & & & & \\
\hline 0.51 & - & Pink & & & & & \\
\hline 0.58 & Green & Pink & & & & & \\
\hline 0.62 & - & Pink & Amino acid & & & & \\
\hline 0.69 & - & Light green & & & & & \\
\hline 0.89 & - & Blue & Nimbin & & & & \\
\hline
\end{tabular}

Table: 3Benzene extract

\begin{tabular}{|c|c|c|c|c|c|c|c|}
\hline \multicolumn{4}{|c|}{ Solvent: Hexane-Ethyl acetate $(5: 5)$} & \multicolumn{4}{|c|}{ Solvent: Methanol- Toluene(8:2) } \\
\hline \multicolumn{4}{|c|}{ Material } & \multicolumn{4}{|c|}{ Material } \\
\hline Rf & Visible light & UV light & $\begin{array}{l}\text { Compound } \\
\text { identified }\end{array}$ & $\mathrm{Rf}$ & Visible light & UV light & $\begin{array}{c}\text { Compound } \\
\text { identified }\end{array}$ \\
\hline 0.089 & - & Blue & Nimbin & 0.46 & Light green & Pink & Nimbin \\
\hline 0.10 & - & - & & 0.50 & Light green & Pink & Nimbin \\
\hline 0.15 & - & - & & 0.61 & - & - & \\
\hline 0.28 & Light green & Dark & & 0.70 & - & - & \\
\hline 0.32 & Light green & Pink & & 0.79 & - & - & \\
\hline 0.48 & Light green & Pink & & & & & \\
\hline 0.64 & - & Pink & Amino acid & & & & \\
\hline 0.71 & - & Light Green & Azadirachtin & & & & \\
\hline 0.90 & - & Blue & Nimbin & & & & \\
\hline
\end{tabular}


Table: 4Ethyl acetate extract

\begin{tabular}{|c|c|c|c|c|c|c|c|}
\hline \multicolumn{4}{|c|}{ Solvent: Hexane-Ethyl acetate(5:5) } & \multicolumn{5}{c|}{ Solvent: Methanol- Toluene(8:2) } \\
\hline \multicolumn{3}{|c|}{ Material } & \multicolumn{3}{c|}{ Material } \\
\hline Rf & Visible light & UV light & $\begin{array}{c}\text { Compound } \\
\text { identified }\end{array}$ & Rf & Visible light & UV light & $\begin{array}{c}\text { Compound } \\
\text { identified }\end{array}$ \\
\hline 0.048 & - & - & & 0.38 & Light green & Pink & \\
\hline $\mathbf{0 . 0 8 8}$ & - & Blue & Nimbin & $\mathbf{0 . 4 3}$ & Light green & Pink & Nimbin \\
\hline 0.193 & - & Pink & & $\mathbf{0 . 5 1 6}$ & Green & Pink & Nimbin \\
\hline 0.258 & Light green & dark & & 0.70 & - & - & \\
\hline 0.403 & Light green & Pink & & 0.85 & - & & \\
\hline 0.50 & Light green & Pink & & & & & \\
\hline 0.5322 & Light green & Pink & & & & & \\
\hline 0.580 & - & Pink & & & & & \\
\hline $\mathbf{0 . 6 2 1}$ & Green & Pink & Amino acid & & & & \\
\hline $\mathbf{0 . 7 1}$ & Light green & Blue & Azadirachtin & & & & \\
\hline $\mathbf{0 . 8 9}$ & - & Blue & Nimbin & & & & \\
\hline
\end{tabular}

Table: 5Ethanol extract

\begin{tabular}{|c|c|c|c|c|c|c|c|}
\hline \multicolumn{5}{|c|}{ Solvent: Hexane-Ethyl acetate (5:5) } & \multicolumn{4}{c|}{ Solvent: Methanol- Toluene(8:2) } \\
\hline \multicolumn{3}{|c|}{ Material } & \multicolumn{4}{c|}{ Material } \\
\hline Rf & Visible light & UV light & $\begin{array}{c}\text { Compound } \\
\text { identified }\end{array}$ & Rf & Visible light & UV light & $\begin{array}{c}\text { Compound } \\
\text { identified }\end{array}$ \\
\hline $\mathbf{0 . 0 8 9}$ & - & Blue & Nimbin & $\mathbf{0 . 4 7 5}$ & Light green & Pink & Nimbin \\
\hline 0.179 & - & - & & $\mathbf{0 . 5 4 0}$ & Light green & Pink & Nimbin \\
\hline 0.2686 & - & Pink & & 0.655 & - & Dark & \\
\hline 0.358 & Light green & Dark & & 0.7377 & - & - & \\
\hline 0.4177 & Light green & Pink & & 0.8196 & - & - & \\
\hline 0.5522 & Light green & Pink & & & & & \\
\hline $\mathbf{0 . 5 9 2 0}$ & Light green & Pink & Amino acid & & & & \\
\hline 0.6417 & - & Dark & & & & & \\
\hline 0.6865 & Green & Pink & & & & & \\
\hline $\mathbf{0 . 7 3 9}$ & - & Pink & Ascorbic acid & & & & \\
\hline $\mathbf{0 . 9 0}$ & - & Blue & Nimbin & & & & \\
\hline
\end{tabular}

Table: 6Toluene extract

\begin{tabular}{|c|c|c|c|c|c|c|c|}
\hline \multicolumn{5}{|c|}{ Solvent: Hexane-Ethyl acetate (5:5) } & \multicolumn{5}{c|}{ Solvent: Methanol- Toluene (8:2) } \\
\hline Rf & Visible light & UV light & $\begin{array}{c}\text { Compound } \\
\text { identified }\end{array}$ & Rf & Visible light & UV light & $\begin{array}{c}\text { Compound } \\
\text { identified }\end{array}$ \\
\hline 0.064 & - & - & & $\mathbf{0 . 4 5 7}$ & Light green & Pink & Nimbin \\
\hline $\mathbf{0 . 0 9 6}$ & - & Blue & Nimbin & $\mathbf{0 . 5 0 8 4}$ & Light green & Pink & Nimbin \\
\hline 0.177 & - & - & & 0.593 & - & Dark & \\
\hline 0.258 & Light green & dark & & 0.644 & - & - & \\
\hline 0.338 & Light green & Pink & & 0.7796 & - & - & \\
\hline 0.435 & Light green & Pink & & & & & \\
\hline 0.532 & Light green & Pink & & & & & \\
\hline $\mathbf{0 . 6 1 2}$ & - & Pink & Amino acid & & & & \\
\hline $\mathbf{0 . 7 0 9}$ & - & Light green & Azadirachtin & & & & \\
\hline $\mathbf{0 . 9 0}$ & - & Blue & Nimbin & & & & \\
\hline
\end{tabular}




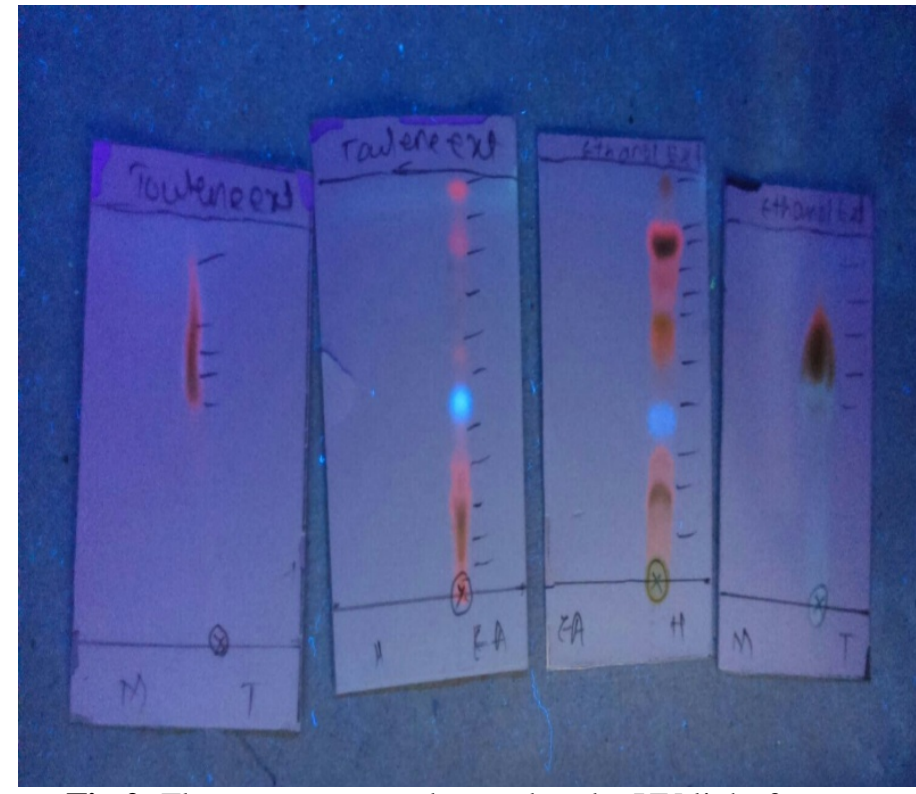

Fig.2: Fluorescent spots observed under UV light for toluene and ethanol extract

With solvent system hexane: ethyl acetate (5:5) and methanol: toluene (8:2).

\section{Techno-Economics:}

Economics of this work is completely based on cost of solvent which is utilized for extraction of compounds from Azadirachta indica leaves and cost for analysis through thin layer chromatography. Solvent can be concluded as best solvent if cost of that particular solvent is less and should be able to extract maximum number compounds present in Azadirachta indica leaves. In this way we can say that particular solvent is economical for further use over industries.

\section{CONCLUSION:}

It is investigated from above result that each and every solvent has own capacity to extract different compounds present in Azadirachta indica leaves which can be utilized for number of medicinal purposes. From the above data we can say that alcoholic solvents are most effective throughout the study since they are able to extract maximum number of compounds present in Azadirachta indica leaves.

Therefore from foregoing discussion, it can be concluded that Azadirachta indica, a common medicinal plant can be exploited as a source of potent biocide since it contains large number of compounds which can be used for large number of applications over all types of industries especially for medicinal purposes.

In the present study, Thin layer chromatographic separation of solvent extracts of plant material contain large number of compounds revealed by fluorescent spots when visualized under UV light as seen in figure 2. The capacity of particular solvent to extract the compounds present in Azadirachta indica leaves is as follows,

Benzene $<$ Toluene $<$ Ethyl acetate $<$ Alcohol

\section{ACKNOWLEDGEMENT:}

All the authors are very much thankful to Lalitha college of pharmacy and Anurag group of Institutions, Hyderabad for their help and cooperation throughout the research work. This research work is done at well equipped labs of Anurag group of Institutions.

\section{REFERENCES:}

[1]. Mondali, N. K., Mojumdar, A., Chatterje. S. K., Banerjee, A., Datta, J.K., Gupta, S. Antifungal activities and chemical characterization of Azadirachta indica leaf extracts on the growth of some selected fungal species in vitro culture medium, J. Appl. Sci. Environ. Manage. March(2009), vol. 13(1), 49 - 53.

[2]. Goutam Brahmachari Neem -An Omnipotent Plant: A Retrospection. Chem Bio Chem (Wiley- VCH), (2004). Vol 5(4), 408-421.

[3]. Bipin D Lade., Anita S Patil., Hariprasad M Paikrao., Ankit S Kale., Kushal K Hire.,A comprehensive working, principles and applications of thin layer chromatography, Research journal of pharmaceutical, biological and chemical sciences.(2014). Vol 5(4), 486-503.

[4]. Pandey Ravindra., Shukla Shankar Shiv., Saraf Warnlata. S., and Saraf Shailendra. Standardization and Validated High-Performance Thin-Layer Chromatographic Fingerprint Method for Quantitative Determination of Plumbagin in a Traditional Indian Formulation. Journal of Planar Chromatography. (2013). Vol.26 (5), 440-444.

[5]. Maitland. P. D and Maitland.D. P. Chromatography: Are we getting it right?. Journal of Biological Education. (2010). 37; 1, 6-8.

[6]. Prus. W and Kowalska.T. Optimization of Thin-Layer Chromatography. Encyclopedia of Chromatography.(Ed.j.Cazes),Marcel Dekker, INC.,New York,2001; Chapter 205, 576-579.

[7]. Cserhati. T and Forgacs. E. Thin-Layer Chromatography of Synthetic Dyes. Encyclopedia of Chromatography. (2007).

[8]. Beyerinck. M. W., Z. Phys. Chem. 3 (1889), 110.

[9]. Issaq. H. J. Recent Developments in Thin-Layer Chromatography - II. Journal of Liquid Chromatography. (2006). 4;6. 955-975.

[10]. Shaw. P. D., G.Ping, S.L.Daly, C. Cha, J. E. Cronan, Jr., K.L. Rinehart., and S. K. Farrand. Detecting and characterizing n-acyl-homoserine lactone signal molecules by thin-layer chromatography. Proc. Natl. Acad. Sci. USA. (1997), 94: 6036-6041.

[11]. Scott. R. M. The Stationary Phase in Thin Layer Chromatography. Journal of Liquid Chromatography. (2006). 4; $12: 2147-2174$.

[12]. Sherma. J. Sample Preparation for Thin Layer Chromatography. Encyclopedia of Chromatography. (2007).

[13]. Pyka. A Vitamins, Hydrophobic, Analysis by Thin Layer Chromatography. Encyclopedia of Chromatography. (2007).

[14]. Kenyon A.S, Layloff. T and Sherma. J. Rapid 
screening of tuberculosis pharmaceuticals by thin layer chromatography. Journal of Liquid Chromatography \& Related Technologies. (2007). 24; 10. 1479-1490.

[15]. Bhawani. S. A, Ibrahim. M. N. M,. Hashim. O. S. R, Mohammad. A and Hena. S. Thin-layer chromatography of amino acids: a review. Journal of Liquid Chromatography \& Related Technologies. (2012). 35; 11. 1497-1516.

[16]. Amal kumar Ghimarey, Cheng-wu Jin, Bimal kumar Ghimirw, Dong Ha Cho, Antioxidant activity and quantitative estimation of azardichtin and nimbin in azadirchta indica A. juss grown in foot hills of Nepal. African journal of biotechnology vol 8, (2009), 30843091.

[17]. Gangwal.A . Extraction, estimation and thin layer Chromatography of flavonoids: a review. World journal of pharmacy and pharmaceutical sciences. (2013) 2:3 1099-1106.

[18]. Vinocur. B and Altman. A Recent advances in engineering plant tolerance to abiotic stress: achievements and limitations. Current Opinion in Biotechnology. (2005) 16,123-132.

[19]. Bele A. A and Khale. A. An Over View on Thin Layer Chromatography. International Journal of Pharmaceutical Science and Research. (2011), 256257.

[20]. Touchstone. J. C and Levin. S. S. Sample Application in Thin Layer Chromatography. Journal of Liquid Chromatography. (2006). 3; 12. 1853-1863.

[21]. Issaq. H. J Modern Advances in Thin-Layer Chromatography. Separation \& Purification Reviews. (2006), 10(1), 76-116.

[22]. Bipin Deochand Lade, Anita Surendra Patil and Hariprasad Madhukarrao Paikrao. Systematic optimization of TLC for wound induced differential secondary metabolites in passiflora foetida.

[23]. Nicolls JM, Birner J, Forsell P. Passicol, an antibacterial and antifungal agent produced by Passiflora plant species: qualitative and quantitative range of activity. Antimicrobial Agents Chemotherapy. (1973), vol 3(1):110-117. 